\title{
CONTROLE DE Alphitobius diaperinus (Panzer) (Coleoptera: Tenebrionidae) EM INSTALAÇÕES PARA FRANGOS DE CORTE
}

\section{Control of Alphitobius diaperinus (Panzer) (Coleoptera: Tenebrionidae) in broiler facilities}

\author{
SOUZA, L.M. ${ }^{1}$; SILVA, G.S. ${ }^{2}$; BELO, M.A.A. ${ }^{1}$; SOARES, V.E. ${ }^{1}$; COSTA, A.J. ${ }^{3}$ \\ 1 Universidade Camilo Castelo Branco \\ ${ }^{2}$ APTA Regional - Noroeste Paulista - Votuporanga/SP \\ ${ }^{3}$ Universidade Estadual Paulista - UNESP - Jaboticabal \\ Endereço para correspondência: Luciano Melo de Souza: lucianomelos@gmail.com
}

\section{RESUMO}

Em busca de uma nova alternativa para o controle do Alphitobius diaperinus, (Cascudinho) avaliou-se, neste estudo, a ação do Spinosad aplicado em duas concentrações (250 ppm e 400 $\mathrm{ppm})$ e duas dosagens $\left(0,05 \mathrm{~L} / \mathrm{m}^{2}\right.$ e $\left.0,1 \mathrm{~L} / \mathrm{m}^{2}\right)$, em instalações de frango de corte infestadas naturalmente por este coleóptero. Avaliações da infestação foram realizadas por capturas de coleópteros com armadilhas, em intervalos semanais até 49 dias após o tratamento. A partir dos resultados do número de adultos e/ou estágios larvais presentes nos grupos controle e tratados foram calculados os percentuais de eficácia dos tratamentos. O Spinosad, na concentração de $250 \mathrm{ppm}$, aplicado na dose de $0,1 \mathrm{~L} / \mathrm{m}^{2}$, foi considerado ineficaz no controle de cascudinhos, porém na concentração de $400 \mathrm{ppm}$ e dose de $0,05 \mathrm{~L} / \mathrm{m}^{2}$ apresentou elevada eficácia e período residual curto. A dose de $0,1 \mathrm{~L} / \mathrm{m}^{2}$ de Spinosad na concentração de $400 \mathrm{ppm}$ proporcionou entre os tratamentos a maior eficácia contra os coleópteros e foi o único tratamento que apresentou, já no sétimo dia pós-tratamento, $100 \%$ de mortalidade das larvas de $A$. diaperinus.

Palavras-chave: avicultura; cascudinho; eficácia; Sacharopolyspora spinosa; Spinosad

\section{ABSTRACT}

Searching for a new alternative to $A$. diaperinus control, it was evaluated the action of Spinosad in two concentrations (250ppm, 400ppm) and two doses $\left(0.05 \mathrm{~L} / \mathrm{m}^{2}\right.$ and $\left.0.1 \mathrm{~L} / \mathrm{m}^{2}\right)$, applied in poultry broiler facilities naturally infested by this coleoptera. Assessments of the infestation were held in weekly intervals, during 49 days after treatment, using traps. The percentage of effectiveness were calculated from the results of the number of adults and/or larval stages in control and treated groups. Spinosad at the concentration of $250 \mathrm{ppm}$, applied at a dose of $0.1 \mathrm{~L} / \mathrm{m}^{2}$, can be considered ineffective against these beetles, however the application of $400 \mathrm{ppm}$ at a dose of $0.1 \mathrm{~L} / \mathrm{m}^{2}$ showed high efficacy and short residual period. The dose of $0.1 \mathrm{~L} / \mathrm{m}^{2}$ of Spinosad at the concentration of 400ppm demonstrated, between treatments, better effectiveness against coleopters, reaching efficacy of $100 \%$ against larvae of $A$. diaperinus, observed after the seventh day post-treatment.

Key words: beetles; efficacy; poultry; Sacharopolyspora spinosa; Spinosad 


\section{INTRODUÇÃO}

Com a expansão avícola industrial, o Alphitobius diaperinus, considerado uma praga secundária de grãos armazenados (Legner e Olton, 1970), encontrou nas instalações de aves o habitat ideal para seu desenvolvimento, se apresentando atualmente como uma das principais pragas da avicultura mundial (Arends, 1987; Steelman, 1996; Paiva, 2000; Silva et al., 2007).

Este inseto atua como reservatório de patógenos como Clostridium perfringens (Vittori et al., 2007), Eimeria, Escherichia, Salmonella, Bacillus, Streptococcus, Aspergillus, vírus causadores das doenças de Marek, Gumboro e Newcastle (Safrit e Axtell, 1984), e é considerado hospedeiro intermediário de cestódeos parasitos de aves (Arends, 1987). O fato de constituírem alimento alternativo, principalmente para pintinhos (Matias, 2000), interfere no desenvolvimento das aves causando desuniformidade do lote, além de causarem prejuízos nas instalações, por produzir túneis e orifícios nos equipamentos (Turner, 1986), tornando-se um sério problema para a indústria avícola.

Medidas de controle como o manejo integrado e o uso de produtos químicos, tais como: Piretróides (Bernardi, 2000; Gomes, 2000; Hamm et al., 2006); organofosforados, clorados e carbamatos (Morales, 1991); lactonas macrocíclicas (Miller, 1990), ácido bórico (Dufour et al, 1992), dentre outros, têm sido os meios mais utilizados pelos produtores para reduzir a população dos besouros.

O Spinosad, um produto desenvolvido pela síntese do metabolismo aeróbio do fungo actinomiceto Sacharopolyspora spinosa, presente no solo, faz parte de um novo grupo de acaricidas conhecido como naturalyte, sendo constituído pela mistura de dois metabólitos naturais do fungo, spinosyn $A$ e spinosyn $D$, que apresentam modo de ação diferenciado. Em insetos, produz excitação do sistema nervoso, levando a contrações involuntárias dos músculos, provocando prostração com tremores e paralisia, efeitos relacionados à ativação de receptores nicotínicos (Thompson et al, 1995). Por outro lado, de acordo com Kaufman et al. (2008), apenas o uso do cyfluthrin e do tetrachlorvinphos são aprovados para o controle do $A$. diaperinus em aviários nos Estados Unidos, apesar de terem sido relatados casos de cepas deste coleóptero resistentes a estes princípios ativos (Hamm et al., 2006)

Em busca de uma nova alternativa para o controle deste coleóptero avaliou-se, neste estudo, a ação do Spinosad, suspensão concentrada (SC), em duas concentrações (250ppm e 400ppm) e em duas dosagens $\left(0,05 \mathrm{~L} / \mathrm{m}^{2}\right.$ e $\left.0,1 \mathrm{~L} / \mathrm{m}^{2}\right)$, aplicadas em instalações avícolas de corte infestadas naturalmente por Alphitobius diaperinus (cascudinhos).

\section{MATERIAL E MÉTODOS}

O estudo foi conduzido em cinco galpões $\left(1500 \mathrm{~m}^{2}\right.$, com dimensões de $12 \mathrm{~m}$ $x 125 \mathrm{~m}$ ) de frangos de corte pertencentes a uma empresa avícola situada no município de Votuporanga, Estado de São Paulo, Brasil. A seleção dos galpões se deu de acordo com o grau de infestação por $A$. diaperinus, sendo os tratamentos estabelecidos aleatoriamente, utilizando-se um galpão para cada grupo experimental, constituindo os seguintes tratamentos: S250D0,1L, S400D0,05L e S400D0,1L, tratados com Spinosad (Eli Lilly do Brasil Ltda.) na concentração de 250 ppm e dose de $0,10 \mathrm{~L} / \mathrm{m}^{2}, 400$ ppm e dose de $0,05 \mathrm{~L} / \mathrm{m}^{2}$ e $400 \mathrm{ppm}$ e dose de $0,10 \mathrm{~L} / \mathrm{m}^{2}$, respectivamente; um grupo controle positivo C300D0,05L tratado com Cyfluthrin (Solfac CE 5 - Bayer S.A. - Saúde Animal) na concentração de 300 ppm e dose de $0,05 \mathrm{~L} / \mathrm{m}^{2}$; um grupo controle negativo $\mathrm{A} 0,05 \mathrm{~L}$ tratamento realizado com água. Dentro de cada tratamento, foram realizados 10 pontos de avaliação. Os 
pontos de avaliação foram tomados como repetições para análise statística.

A aplicação dos medicamentos, no dia zero, foi realizada, com auxílio de bomba costal, via aspersão, nas muretas (faces interna e externa), postes, piso e calçadas laterais de cada aviário; durante a medicação as cortinas foram mantidas fechadas. Um dia após os tratamentos em cada ponto de avaliação procedeu-se a contagem de $A$. diaperinus mortos ou em estado de paralisia. Adultos foram considerados em estado de excitação/tetania quando, no momento da avaliação, não conseguiam se locomover, porém, apresentavam-se em posição de decúbito dorsal com movimentos de patas e/ou antenas. Para os estágios larvais consideraram-se, também, os que não se locomoveram normalmente, em sua maioria na posição decúbito dorsal, e com movimentos de patas. Foram considerados mortos aqueles que se apresentaram sem qualquer movimento e enrijecidos (adultos e larvas). Os instares considerados mortos foram alocados em placas de petri separadas e identificadas pelo seu grupo de origem, sendo observados quanto aos sinais vitais por mais duas semanas.

Após o período de vazio sanitário, as instalações receberam cama de casca de arroz onde foram alojados pintos de um dia de idade da linhagem COBB na mesma densidade por galpão. Avaliações da infestação por $A$. diaperinus em cada galpão foram realizadas em intervalos semanais, sendo uma anterior ao início do tratamento (AT) seguidas por mais sete até $0 \quad 49^{\circ}$ dia após o tratamento, coincidindo 0 encerramento do experimento com o final do ciclo de produção dos lotes. Para as avaliações, armadilhas seguindo 0 modelo modificado de Arend's (Stringhan e Arends, 1990) foram colocadas em 10 pontos dentro de cada galpão. Em intervalos semanais, o conteúdo de cada armadilha era recolhido e o número de $A$. diaperinus contado.

A partir dos resultados do número de adultos e/ou estágios larvais, vivos e com movimentos normais, presentes nos grupos controle e tratados, foram calculados os percentuais de eficácia dos tratamentos, em cada data avaliada de acordo com a fórmula abaixo:

$\%$ Eficácia $=\frac{A-B}{A} \times 100$, onde:

A - Número de indivíduos vivos no grupo controle negativo;

B - Número de indivíduos vivos no grupo tratado;

Foi utilizado um delineamento em parcela subdividida no tempo ("Split Plot in Time") sendo considerado, como tratamento principal, as aplicações dos fármacos (concentrações/doses) e o controle, e como secundário as datas de observações (Banzatto e Kronka, 1989). As análises dos dados foram realizadas aplicando-se o teste $\mathrm{F}$ e as médias (dados transformados em log $(\mathrm{x}+1)$ ) comparadas pelo teste de Tukey (SAS, 1989).

\section{RESULTADOS E DISCUSSÃO}

Antes do estabelecimento dos tratamentos (AT) todos os galpões experimentais apresentavam-se homogêneos quanto à infestação por $A$. diaperinus. Em nenhuma contagem (Tabela 1), o número de formas adultas de $A$. diaperinus do grupo tratado com $0,10 \mathrm{~L} / \mathrm{m}^{2}$ de Spinosad a 250 ppm foi menor $(P>0,05)$ que a do grupo controle negativo.

Em relação à avaliação da infestação por larvas, houve o mesmo comportamento, com exceção no $35^{\circ}$ dia após o tratamento (DPT), pois o tratamento com $0,10 \mathrm{~L} / \mathrm{m}^{2}$ de Spinosad a 250ppm resultou em percentuais de eficácia abaixo de $80 \%$, o que caracteriza baixa eficácia no controle deste coleóptero (Tabela 1). Em estudo realizado por Kaufman et al. (2008), o uso do Cyfluthrin e do Tetraclorvinphos apresen- 


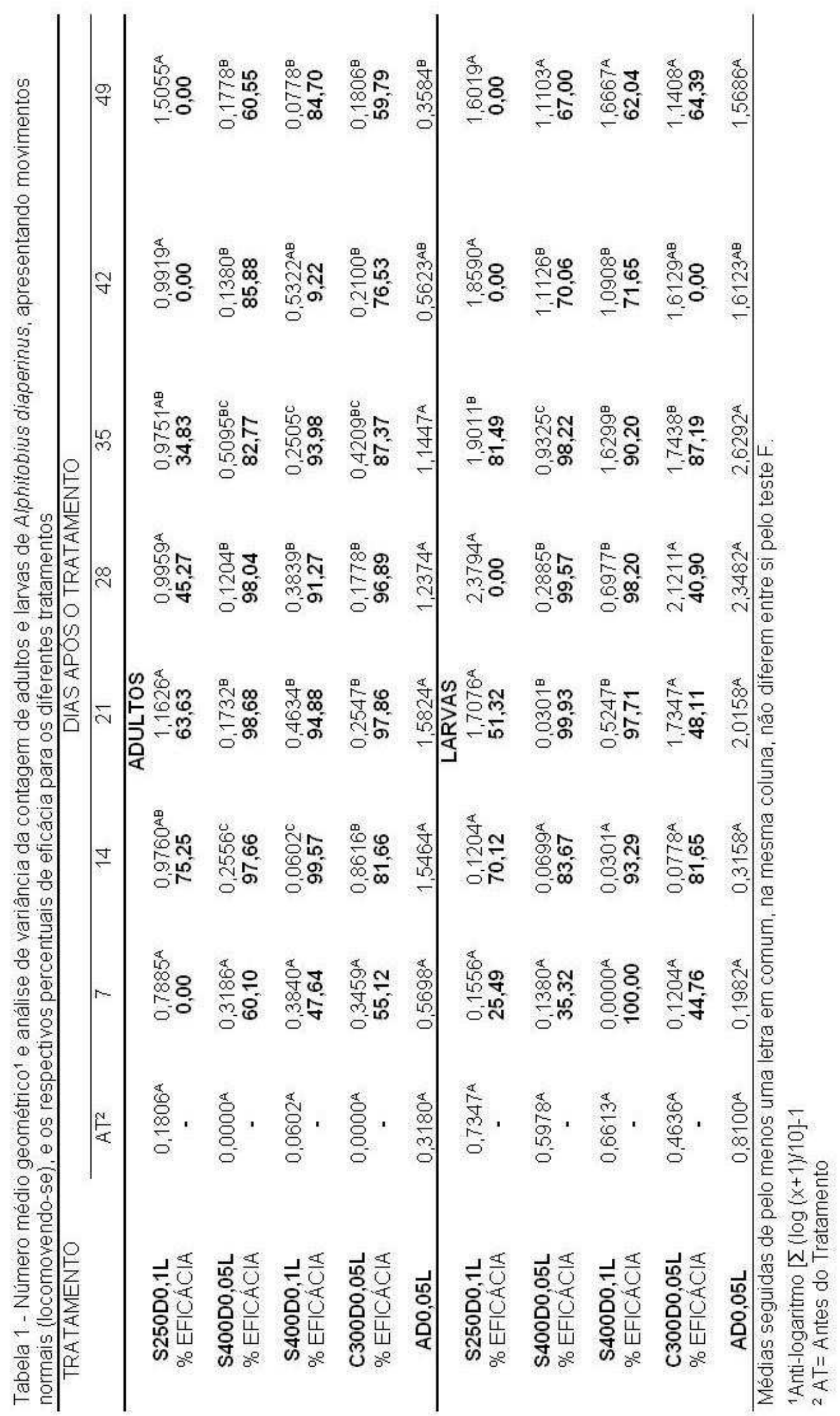


tou baixa eficácia no controle de algumas cepas de $A$. diaperinus. Para estes autores, tal fato indica 0 desenvolvimento de resistência, prejudicando o manejo sanitário dos aviários.

Por outro lado, a concentração de 400 ppm, na sua menor dosagem $(0,05$ $\left.\mathrm{L} / \mathrm{m}^{2}\right)$, contra adultos, foi superior a $95 \%$ no período do $14^{\circ}$ ao $28^{\circ} \mathrm{DPT}(97,66 \%$, $98,68 \%$ e $98,04 \%$, respectivamente). Contra larvas, a atuação foi acima de $98,00 \%$ no intervalo de $21^{\circ}$ ao $35^{\circ}$ DPT (99,93\%, 99,57\% e 98,22\% de eficácia, Tabela 1).

Para o controle efetivo da infestação por $A$. diaperinus em avicultura, é desejável que a eficácia do fármaco utilizado seja mantida por um período que se aproxime ao tempo de estadia das aves nas instalações, ou seja, do seu ciclo de produção. Assim, a maior persistência de eficácia ocorreu com a aplicação do Spinosad a $0,10 \mathrm{~L} / \mathrm{m}^{2}$ na concentração de 400 ppm, em que o período de ação foi mais longo contra adultos, resultando entre o $14^{\circ}$ ao $35^{\circ}$ DPT, numa mortalidade de $99,57 \%$, $94,88 \%, 91,27 \%$ e $93,98 \%$. Contra larvas, o intervalo foi maior, do $7^{\circ}$ ao $35^{\circ}$ DPT ocorreu mortalidade de 100,00\%, $93,29 \%, 97,71 \%, 98,20 \%$ e $90,20 \%$, respectivamente. Chama-se atenção, que somente neste tratamento ocorreu, já no sétimo dia após o tratamento, uma eficácia de $100 \%$ contra larvas de cascudinho. Os Índices de eficácia foram semelhantes aos observados por Morales (1991) que verificou baixo efeito biocida in vitro dos produtos malathion, carbaril e dipterex quando aplicados a $0,5 \mathrm{~L} / \mathrm{m}^{2}$ e $100 \%$ de mortalidade dos coleópteros, quando elevou-se a aplicação para 1 $\mathrm{L} / \mathrm{m}^{2}$.

Os resultados de eficácia neste estudo apresentam-se compatíveis com os publicados por Weaver (1996) que observou elevada eficácia contra $A$. diaperinus com uso de reguladores de crescimento de insetos (IGR) e piretróides aplicados em instalações de frangos de corte, observando $95-100 \%$ de controle de larvas no período de 42 dias. Ainda, Khan et al (1998) observaram adequado controle de larvas e adultos de $A$. diaperinus em até cinco semanas da aplicação de Tetrachorvinphos em instalações de frangos de corte. Silva et al. (2007) verificaram eficácia de $100 \%$ no controle de larvas e adultos $A$. diaperinus após o tratamento de cama de frango com a associação de cipermetrina (15\%), clorpirifós $(25 \%)$ e citronela $(1 \%)$.

O Cyfluthrin atingiu um percentual satisfatório de $97,86 \%$ e $96,89 \%$ na mortalidade de adultos nos dias 21 e 28 DPT, respectivamente (Tabela 1). Porém, contra larvas a eficácia manteve-se inferior a 90\%. Tais achados estão em concordância aos descritos por Hamm et al. (2006) que observaram resistência de cinco cepas diferentes de $A$. diaperinus provenientes de instalações avícolas, submetidas ao tratamento químico com Cyfluthrin.

Não foi possível observar correlação entre a mortalidade imediata dos coleópteros e o período de ação residual dos fármacos como descrito em outros estudos (Tomberlin et al., 2008). Na Tabela 2 , observou-se mortalidade $(P<0,05)$ maior no grupo tratado com Cyfluthrin em relação aos tratamentos com Spinosad. No entanto, o uso indiscriminado deste piretróide no controle do $A$. diaperinus nos Estados Unidos resultou em elevado nível de resistência (Hamm et al., 2006). Por outro lado, Lambkin e Rice (2007) demonstraram a eficácia inseticida do Spinosad em 13 cepas de $A$. diaperinus provenientes de aviários de frango de corte na Austrália e resistentes a diferentes inseticidas, inclusive ao Cyfluthrin. Estes autores discutem em seus estudos a possibilidade da ocorrência de resistência cruzada deste coleóptero aos diferentes inseticidas. Porém, este efeito não foi observado por estes autores na comparação entre o tratamento de Cyfluthrin e Spinosad, provavelmente, devido à diferença na farmacodinâmica destes 
compostos. As observações e discussões geradas por estes autores em ambos os estudos refletem a importância da rotação de princípios ativos no controle sanitário deste coleóptero em aviários de frango de corte.

Tabela 2 - Comparação múltipla do número médio e análise de variância da contagem de adultos e larvas de Alphitobius diaperinus mortos

\begin{tabular}{lcc}
\hline \multirow{2}{*}{ TRATAMENTO } & \multicolumn{2}{c}{$\begin{array}{c}\text { № A. diaperinus } \\
\text { MORTOS }\end{array}$} \\
\cline { 2 - 3 } & adultos & larvas \\
\hline Spinosad - $250 \mathrm{ppm}\left(0,10 \mathrm{~L}^{-\mathrm{m}^{2}}\right)$ & $6,80^{\mathrm{B}}$ & $116,20^{\mathrm{C}}$ \\
Spinosad - $400 \mathrm{ppm}\left(0,05 \mathrm{~L} / \mathrm{m}^{2}\right)$ & $9,00^{\mathrm{B}}$ & $345,40^{\mathrm{B}}$ \\
Spinosad - $400 \mathrm{ppm}\left(0,10 \mathrm{~L} / \mathrm{m}^{2}\right)$ & $18,00^{\mathrm{B}}$ & $510,90^{\mathrm{B}}$ \\
Cyfluthrin - $300 \mathrm{ppm}\left(0,05{\left.\mathrm{~L} / \mathrm{m}^{2}\right)}^{2}\right.$ & $41,00^{\mathrm{A}}$ & $720,10^{\mathrm{A}}$ \\
Valor de F & 16,23 & 25,62 \\
Pr>F$^{1}$ & 0,0001 & 0,0001 \\
CV $^{2}$ & 65,63 & 37,75 \\
DMS $^{3}$ & 14,781 & 192,41 \\
\hline
\end{tabular}

Médias seguidas de pelo menos uma letra em comum, na mesma coluna, não diferem entre si pelo teste $\mathrm{F}$.

Probabilidade de significância associada ao valor de $\mathrm{F}$.

CV = Coeficiente de Variação (\%).

DMS: Diferença Mínima Significativa.

Por outro lado, os resultados de mortalidade ao tratamento com Spinosad e Cyfluthrin observados neste estudo (Tabela 2), comprovam a sensibilidade da cepa de $A$. diaperinus estudada a ambos os fármacos testados. Neste sentido, os dados observados no experimento permitem concluir que a aplicação de Spinosad na concentração de $400 \mathrm{ppm}$ e dose de $0,10 \mathrm{~L} / \mathrm{m}^{2}$ é uma alternativa eficaz para controle de cascudinho em aviários para frango de corte.

\section{CONCLUSÕES}

O tratamento com a dose de $0,10 \mathrm{~L} / \mathrm{m} 2$ do Spinosad a $400 \mathrm{ppm}$ proporcionou maior eficácia contra Alphitobius diaperinus e foi o único tratamento que apresentou, já no sétimo dia após o tratamento, eficácia de 100\% contra larvas deste coleóptero, demonstrando ser uma alternativa eficaz para o controle deste coleóptero em aviários, principal- mente, em cepas resistentes ao Cyfluthrin.

\section{REFERÊNCIAS}

ARENDS, J.J. Control, management of the litter beetle. Poultry Digest, v.44, p.172-176, 1987.

BANZATTO, D.A., KRONKA, S.N. Experimentação Agrícola. Jaboticabal, FUNEP, 1989.

BERNARDI, P.L. Programa para controle do cascudinho (Alphitobius diaperinus). In: Simpósio Brasil Sul de Avicultura. Anais... p.153-157, 2000.

DUFOUR, L., SANDER, J.E.; WYATT, R.D. et al. Experimental exposure of broiler chickens to boric acid to assess clinical signs and lesion of toxicosis. Avian Diseases, v.36, p.1007-1011, 1992.

GOMES, J.P.C. Controle de Alphitobius diaperinus (Panzer) (Coleoptera: Tenebrionidae) em aviários. In: Simpósio Brasil Sul de Avicultura. Anais... p.143-147, 2000.

HAMM, R.L.; KAUFMAN, P.E.; REASOR, C.A. et al. Resistance to cyfluthrin and tetrachlorvinphos in the lesser mealworm, Alphitobius diaperinus, collected from the eastern United States. Pest Management Science, v.62, p.673-677, 2006.

KHAN, B.A.; DAY, P.A.; GOONEWARDENE, L.A. et al. Efficacy of tetrachlorvinphos insecticide dust against darkling beetles in commercial broiler chicken barns. Canadian Journal of Animal Science, v.78, n.4, p.723-725, 1998.

KAUFMAN, P.E.; STRONG, C., RUTZ, D.A. Susceptibility of lesser mealworm (Coleoptera: Tenebrionidae) adults and larvae exposed to two commercial insecticides on unpainted plywood panels. Pest Management Science, v.64, p.108-111, 2008.

LAMBKIN, T.A.; RICE, S.J. Baseline responses of Alphitobius diaperinus (Coleopter: Tenebriionidae) to Spinosad, and susceptibility of broiler populations in Eastern and Southern Australia. Journal of Economic Entomology, v.100, p.1423-1427, 2007.

LEGNER, E.F.; OLTON, G.S. Worldwide survey and comparison of adult predatored scavenger insect populations associated with domestic animal manure where livestock is artificially congregated. Hilgardia, v.40, p.225-56, 1970.

MATIAS, R. S. O controle do cascudinho. Novas perspectivas. In: Simpósio Brasil Sul de Avicultura. Anais... p.169-171, 2000.

MILLER, R.W. Use of ivermectin to control the lesser mealworm (Coleoptera: Tenebrionidae) in a simulated poultry broiler house. Poultry Science, v.69, p.12811284, 1990. 
MORALES, A. Control químico del coleóptero Alphitobius diaperinus com Malathion, Carbaril y Dipterex e granjas avícolas. Revista Cubana de Ciencia Avícola, v.18, p.205-209, 1991.

PAIVA, D.P. Cascudinhos: Biologia. In: Simpósio Brasil Sul de Avicultura. Anais... p.135-39, 2000.

SAFRIT, R.D.; AXTELL, R.C. Evaluations of sampling methods for darkling bettles (Alphitobius diaperinus) in the little of turkey and broiler houses. Poultry Science, v.63, p.2368-75, 1984.

SAS Institute, 1989-1996. SAS ${ }^{\circledR}$ User's Guide: Estatistics. SAS Insnstitute, Inc. Cary, NC, USA.

SILVA, G.S.; MICHELS, M.G.; TOMA, S.B. et al. Effectiveness of the compound Chlorpyriphos + Cypermethrin + Citronella against Alphitobius diaperinus. Laboratory analysis and residue determination in carcasses. Brazilian Journal of Poultry Science, v.9, n.3, p.167-170, 2007.

STEELMAN, D. Darkling beetles are costly pests. Poultry Digest, v.55, n.10, p.22-23, 1996.

STRINGHAM, M.; ARENDS J.J. Evaluation of a modified Arend's tube trap for sampling the lesser mealworm, Alphitobius diaperinus (Panzer), in turkey brooder houses. In: Livestock Insect Workers Conference (não publicado), 1990.
TOMBERLIN, J.K.; RICHMAN, D.; MYERS, H. Susceptibility of Alphitobius diaperinus (Coleoptera: Tenebrionidae) from broiler facilities in Texas to four insecticides. Journal of Economic Entomology, v.101, p.480-483, 2008.

THOMPSON, G.D.; BUSACCA, J.D.; JANTZ, O.K. et al. Spinosyns: an overview of new natural insect management systems. In: Beltwide Cotton Production Conference, 1995. Proceedings... Menphis: National Cotton Council, 1995. p.1039-1043.

TURNER JR, E.C. Structural ad litter pests. Poultry Science, v.65, p.644-648, 1986.

VITTORI, J.; ITURRINO, R.P.S.; TROVÓ, K.P. et al. Alphitobius diaperinus como veiculador de Clostridium perfringens em granjas avícolas do interior paulista. Ciência Rural, v.37, n.3, p.894-896, 2007.

WEAVER, J.E. The lesser mealworm, Alphitobius diaperinus: field trials for control in a broiler house with insect growth regulators ad pyrethroids. Journal of Agricultural Entomology, v.13, n.2, p.93-7, 1996. 